\title{
Micromechanical characterization of ferritic ductile cast iron by using instrumented indentation and atomic force microscopy
}

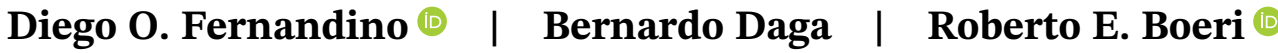

Metallurgy Division, INTEMA CONICET, National University of Mar del Plata, Mar del Plata, Argentina

\section{Correspondence}

Diego O. Fernandino, Metallurgy Division, INTEMA - CONICET, National University of Mar del Plata, 10850 Colón Av., B7606BVZ Mar del Plata, Argentina. Email: dfernandino@fi.mdp.edu.ar

\begin{abstract}
The aim of this investigation is to characterize the mechanical properties at the microstructural level in ferritic ductile iron. The analysis involves microstructural characterization, nanoindentation testing, atomic force microscopy analysis, and the application of an inverse algorithms proposed in the literature.

The results show that, because of microsegregation, different regions of a single-phase ferritic matrix have different elastic-plastic behavior. The methodology developed in this work becomes useful to evaluate the mechanical properties along the metallic matrix of other ductile iron microstructures.

\section{KE YWO R D S}

atomic force microscopy, ductile iron, elasto-plastic indentation, mechanical properties, microsegregation
\end{abstract}

\section{1 | INTRODUCTION}

Ferritic ductile iron (FDI) is a broadly used DI grade, consisting in a continuous ferritic metallic matrix, with a dispersion of graphite nodules. It can be obtained as-cast or after an annealing heat treatment. It is used when ductility and good impact properties are required. Trucks and agricultural implements, automotive parts, valves, and fittings, among others, are the common applications. ${ }^{1}$

Due to its heterogeneous nature, the microstructural characterization of DI needs geometric and constitutive behavior data of the material. The geometrical characterization is typically given by the volume fraction of graphite, nodularity, and nodule count. In general, higher nodular count promotes better mechanical properties. ${ }^{1}$ Regarding the constitutive behavior of the material, the earlier assumption was that the DI had a homogeneous matrix. ${ }^{1-5}$ However, the experimental evidence shows that the metallic matrix has a high degree of heterogeneity, as a result the segregation process taking place during solidification. The first-to-freeze (FTF) zones, coincident with the axes and arms of the austenite dendrites, and the last-to-freeze (LTF) zones, coincident with the last portions of the remaining melt located between them, are identified. ${ }^{6}$ In the last years, several works regarding the microsegregated LTF and FTF zones were conducted by different authors. ${ }^{7-10}$ But the influence of these heterogeneities on the mechanical properties at the microstructural level received little attention. Therefore, it is necessary to develop an experimental analysis to assess these heterogeneities and analyze their influence on the mechanical properties at the microstructural level. 
In this context, instrumented indentation tests emerge as a powerful tool to determine the mechanical properties at the microstructural level. During the last 20 years, different approaches were proposed. Most of them are referring to the possibility of finding more than one solid that essentially exhibits the same contact behavior. That is, similar load versus displacement curves and the uniqueness of the solution provided by inverse methods were discussed. ${ }^{11-16}$

In this work, the uniaxial mechanical properties at the microstructural level in ferritic DI is characterized. The analysis involves microstructural characterization, nanoindentation testing, atomic force microscopy analysis, and the application of an inverse algorithm proposed by Mata and Alcala. ${ }^{11}$

\section{2 | MATERIALS AND METHODS}

\section{1 | Cast material and metallographic characterization}

The DI samples were obtained from $25 \mathrm{~mm}$ "Y" blocks cast in sand molds, according to ASTM A897M standard. The chemical compositions of the melts were determined by using a Baird DV6 spectrometer. Aiming to achieve a fully ferritic metallic matrix, the $25 \mathrm{~mm}$ "Y" blocks were ferritized by a heat treatment consisting of an austenitizing stage at $920^{\circ} \mathrm{C}$ for $1 \mathrm{~h}$ followed by a slow cooling down to room temperature inside the furnace. The metallographic samples were prepared by means of standard polishing and etching methods. Etching was carried out employing Nital (2\%) and Motz reagent. ${ }^{17}$ The Motz reagent is sensitive to the segregation of $\mathrm{Si}$, and it is usually employed to locate the LTF in DI microstructures. For all cases, the microstructural characterization was done by using an optical microscope OLYMPUS PMG3 and the Image-Pro Plus software.

\section{2 | Uniaxial mechanical properties estimation}

The uniaxial mechanical properties were estimate from the methodology proposed by Mata and Alcala. ${ }^{11}$ They used mathematical formulations to correlate the hardness and the amount of pileup and sinking-in phenomena around sharp indenters with the uniaxial mechanical properties. The main parameters involved in the formulation are the hardness, $H$, and $\alpha$, which is defined as follows:

$$
\sqrt{\alpha}=\frac{h}{h s},
$$

where $h s$ denotes the depth of penetration from the center of the indentation to the free (undeformed) surface of the solid and $h$ is the depth of penetration with respect to the true contact perimeter of deformed surface. Therefore, $h$ accounts for the actual surface deformation in the material so that $\sqrt{\alpha}>1$ implies the development of pileup and $\sqrt{\alpha}<1$ indicates sinking-in. According to Mata and Alcala, ${ }^{11}$ the formulations proposed are applicable regardless of the ruling deformation regime of a strain-hardening solid. Using the inverse algorithm proposed, the yield strength $\left(\sigma_{y s}\right)$ and the hardening coefficient $(n)$ can be estimated from the Young modulus $(E)$, hardness $(H)$, and the $\alpha$ parameter. Details can be found in Mata and Alcala. ${ }^{11}$

\section{3 | Instrumented indentation and AFM tests}

The instrumented indentation tests were performed using a T1900 SERIES Hysitron equipped with the Multirange nanoprobe "MRNP" and Berkovich indenter. The indentations were done in rectangular patterns. All tests were performed in loading control mode with a maximum load of 95,020 $\mu \mathrm{N}$. At least 25 indentations over each analysis zone were conducted. The $E$ and $H$ values were retrieved from the instrumented indentation testing by means of the Oliver and Pharr approach. ${ }^{18}$ Then, indentations were scanned by using an Atomic Force Microscope Agilent technologies 5500. Aiming to calculate the $\alpha$ parameter, data were postprocessed using Gwyddion ${ }^{\circledast}$ software. $^{2}$ 


\section{3 | RESULTS}

\section{1 | Material characterization}

The chemical composition presents $3.32 \% \mathrm{C}, 2.36 \% \mathrm{Si}, 0.31 \% \mathrm{Mn}, 0.033 \% \mathrm{Mg}, 0.62 \% \mathrm{Cu}, 0.012 \% \mathrm{~S}$, and $0.016 \% \mathrm{P}(\%$ weight), which corresponds to a slightly hypoeutectic DI, including small amounts of $\mathrm{Cu}$ and $\mathrm{Mn}$. The microstructure resulting from the annealing heat treatment and the subsequent etching with Nital (2\%) and Motz are shown in Figure 1. A fully ferritic matrix is shown in Figure 1A. However, after applying the Motz etching, the heterogeneity within the ferrite is revealed, in which the FTF and the LTF zones are identified, as shown in Figure 1B,C. Note that the LTF zones are delimited by a dark boundary and they show small black dots consistent with precipitates or microvoids.

\section{2 | Uniaxial mechanical properties determination}

The relation between load $(P)$ versus displacement $(h)$ retrieved from the instrumented indentation testing on both FTF and LTF zones are plotted in Figure 2A,B, respectively. The experimental dispersion of data is represented by a double curve in each plot. Naturally, the LTF zones show a higher dispersion due to the heterogeneities involved, as was identified in Figure 1C. The postprocessing of the AFM data of one indentation is schematized in Figure 3. A minimum of

FIG URE 1 Optical metallography resulting from etching with different reagent. (A) Nital 2\%, (B) Motz-50×, (C) Motz-200x

F I G U RE $2 \operatorname{Load}(P)$ vs displacement $(h)$ curves retrieved from the instrumented indentation testing by using Berkovich indenter. Double curves are used for representing the experimental dispersion.

(A) FTF, (B) LTF

F I G U RE 3 Postprocessing AFM data of the indentation. (A) Indentation profiles heights, (B) 3D reconstruction of the indentation by using Gwyddion software

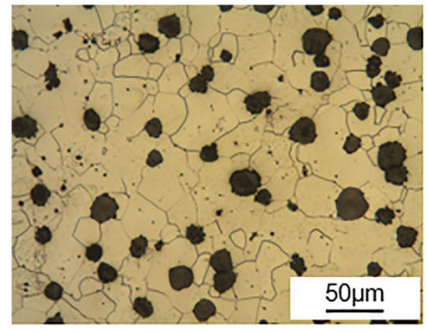

(A)

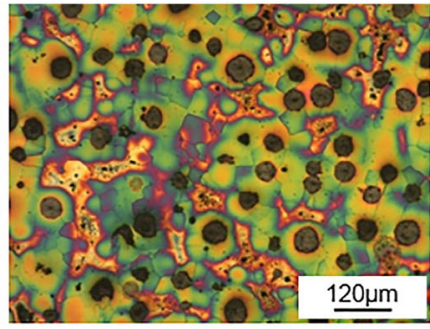

(B)

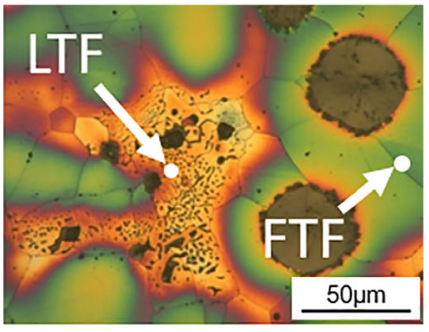

(C)

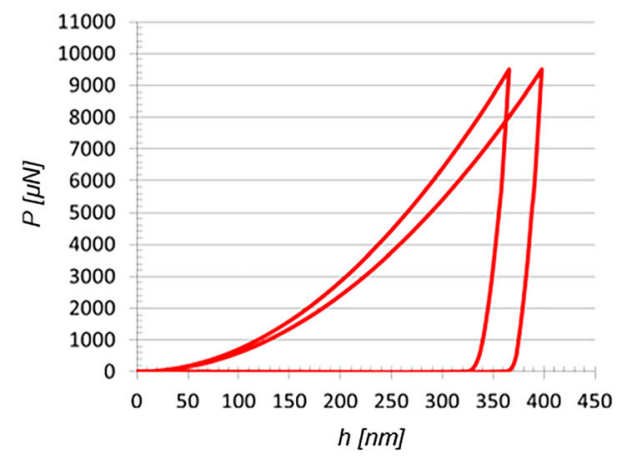

(A)

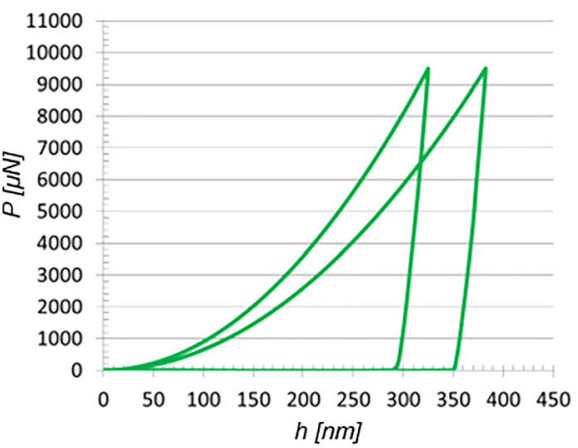

(B)

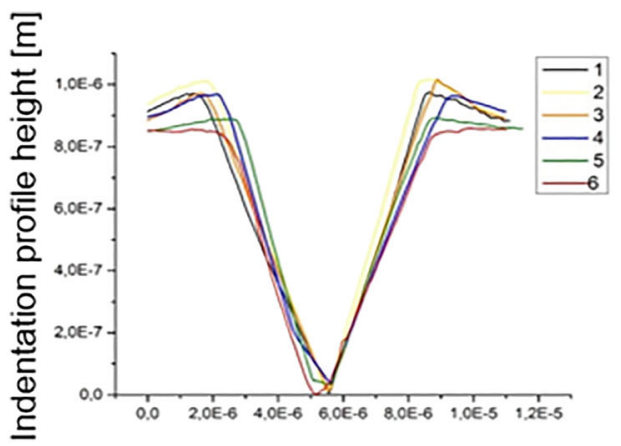

(A)

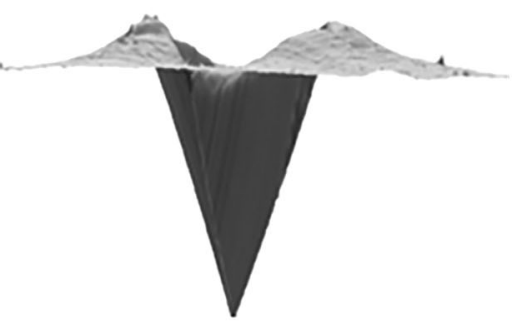

(B) 


\begin{tabular}{llllll|} 
& $\boldsymbol{H}(\mathbf{G P a})$ & $\boldsymbol{E}(\mathbf{G P a})$ & $\sqrt{ } \boldsymbol{\alpha}$ & $\boldsymbol{n}$ & $\boldsymbol{\sigma}_{\boldsymbol{y s}}(\mathbf{M P a})$ \\
\hline FTF zone & $1.65 \pm 0.09$ & $230 \pm 7$ & $1009 \pm 0.001$ & $0.21 \pm 0.01$ & $212 \pm 13$ \\
\hline LTF zone & $2.00 \pm 0.13$ & $250 \pm 8$ & $1100 \pm 0.003$ & $0.21 \pm 0.01$ & $274 \pm 18$ \\
\hline
\end{tabular}

T A B L E 1 Results retrieved from the indentation test, AFM, and the application of inverse algorithm proposed by Mata and Alcala ${ }^{11}$

12 profile in different directions of each indentation mark was determined. Figure 3B shows the plot of the indentation profiles. Those profiles were used to determine the mean pile-up heights and, consequently, the $\sqrt{\alpha}$ value. Figure 3C exposes a 3D reconstruction of an indentation mark in the LTF area. Therefore, the uniaxial mechanical properties were estimated from the inverse analysis proposed by Mata and Alcala. ${ }^{11}$ The results obtained from the instrumented indentation testing, AFM and mechanical properties, are listed in Table 1 for FTF and LTF areas. Therefore, despite both zones show similar elastic properties and the same plastic response (hardening coefficient), the analysis of mechanical properties prove that LTF zones are microstructural zones showing greater strength than the FTF ones.

\section{4 | CONCLUSIONS}

- The indentation analysis shows that the FTF zones and the LTF zones of the FDI have similar elastic behaviors. Consequently, they can be assimilated to a single matrix material, in accordance with the usual hypothesis found in the literature.

- The elastoplastic results of LTF zones allow inferring that this area of the metallic matrix has a greater strength than the FTF. The hardness shows values $20 \%$ higher than the FTF zones, while the yield strength shows values 30\% higher. Anyways, the hardening coefficients of both segregated regions show the same value.

- The experimental methodology used in this work becomes useful to evaluate the mechanical properties along the matrix of other DI microstructures.

\section{ORCID}

Diego O. Fernandino (1) https://orcid.org/0000-0003-4647-2663

Roberto E. Boeri (10 https://orcid.org/0000-0001-7083-579X

\section{REFERENCES}

1. Ductile Iron Society. Ductile Iron Data for Design Engineers. Rio Tinto Iron \& Titanium Inc; 1998.

2. Carazo FD, Giusti SM, Boccardo D, Godoy L. Effective properties of nodular cast-iron: a multi-scale computational approach. Comput Mater Sci. 2014;82:378-390.

3. Basso A, Martinez R, Cisilino A, Sikora J. Experimental and numerical assessment of fracture toughness of dual-phase austempered ductile iron. Fatigue Fract Eng Mater Struct. 2009;33:1-11.

4. Bonora N, Ruggiero A. Micromechanical modeling of ductile cast iron incorporating damage. Part I: ferritic ductile cast iron. Int J Solid Struct. 2005;42:1401-1424.

5. Ortiz J, Cisilino AP, Otegui JL. Effect of microcracking on the micromechanics of fatigue crack growth in austempered ductile iron. Fatigue Fract Eng Mater Struct. 2001a;4302:591-605.

6. Rivera G, Boeri R, Sikora J. Revealing and characterizing solidification structure of ductile cast iron. Mater Sci Technol. 2002;18:691-698.

7. Fernandino D, Tenaglia N, Di Cocco V, Boeri R, Iacoviello F. Relation between microstructural heterogeneities and damage mechanisms of a ferritic spheroidal graphite cast iron during tensile loading. Fatigue Fract Eng Mater Struct. 2020;43:1262-1273.

8. Fernandino DO, Boeri RE. In-situ microscopic analysis of ferritic ductile iron during tensile loading: relation between matrix heterogeneities and damage mechanisms. Fatigue Fract Eng Mater Struct. 2019;42:2220-2231.

9. Fernandino D, Cisilino A, Toro S, Sánchez P. Multi-scale analysis of the early damage mechanics of ferritic ductile iron. Int $J$ Fract. 2017;207:1-26.

10. Xu C, Andriollo T, Zhang Y, Hernando J, Hattel J, Tiedje N. Micromechanical impact of solidification regions in ductile iron revealed via a 3D strain partitioning analysis method. Scr Mater. 2020;178:463-467.

11. Mata M, Alcala J. Mechanical property evaluation through sharp indentations in elastoplastic and fully plastic contact regimes. $J$ Mater Res. 2003;17:1705-1709.

12. Mata M, Anglada M, Alcalá J. Contact deformation regimes around sharp indentations and the concept of the characteristic strain. J Mater Res. 2002;17:964-976.

13. Liu L, Ogasawara N, Chiba N, Chen X. Can indentation technique measure unique elastoplastic properties? J Mater Res. 2009;24: 784-800. 
14. Chen X, Ogasawara N, Zhao M, Chiba N. On the uniqueness of measuring elastoplastic properties from indentation: the indistinguishable mystical materials. J Mech Phys Solids. 2007;55:1618-1660.

15. Lan HZ, Venkatesh TA. On the uniqueness and sensitivity issues in determining the elastic and plastic properties of power-law hardening materials through sharp and spherical indentation. Philos Mag a. 2007a;87:4671-4729.

16. Lan HZ, Venkatesh TA. On the sensitivity characteristics in the determination of the elastic and plastic properties of materials through multiple indentation. J Mater Res. 2007b;22:1043-1063.

17. Motz M. Microsegregation, an easily unnoticed influencing variable in the structural description of cast materials. Pract Metallogr. 1988; 25:285-293.

18. Oliver WC, Pharr GM. An improved technique for determining hardness and elastic modulus using load and displacement sensing indentation experiments. Journal of Material Research. 1992;7:1564-1583.

How to cite this article: Fernandino DO, Daga B, Boeri RE. Micromechanical characterization of ferritic ductile cast iron by using instrumented indentation and atomic force microscopy. Mat Design Process Comm. 2020;e206. https://doi.org/10.1002/mdp2.206 